Stud. Univ. Babeş-Bolyai Math. 66(2021), No. 1, 47-53

DOI: $10.24193 /$ subbmath.2021.1.04

\title{
On the existence of a periodic solution of the Liénard system
}

\author{
Alexander Ignatyev
}

Dedicated to Professor Gheorghe Moroşanu on the occasion of his 70th anniversary.

\begin{abstract}
The Liénard system $\frac{d x}{d t}=y, \frac{d y}{d t}=-f(x) y-g(x)$ is considered. Under some assumptions on functions $f(x)$ and $g(x)$, we prove the existence of a periodic solution of this system.
\end{abstract}

Mathematics Subject Classification (2010): 34C05, 34C07, 34C25, 34C15, 34A26.

Keywords: Liénard system, a periodic solution.

\section{Introduction}

On the phase plane to periodic solutions of an autonomous system

$$
\dot{x}=X(x, y), \quad \dot{y}=Y(x, y)
$$

correspond closed trajectories. Such solutions usually describe continuous periodic processes. Periodic solutions are an important class of solutions to ordinary differential equations, since many of the processes described by ordinary differential equations are periodic. A large number of scientific papers are devoted to their study. At his time, Henri Poincaré attached great importance to periodic solutions represented by closed orbits. According to his plans, they were to become a support in the study of all other, non-periodic movements. In a certain sense, periodic solutions are the only type of solutions that can be completely observed in the process of their evolution, since the entire evolution of a periodic solution is determined by the knowledge of this solution over a finite period of time. Periodic solutions are the simplest type of oscillatory solutions.

In 1928, Liénard [7, 8] considered equations of the form

$$
\frac{d^{2} x}{d t^{2}}+f(x) \frac{d x}{d t}+x=0
$$


where $f(x)$ is a polynomial of even degree. These equations arose as a generalization of the famous van Der Pol equation [12]

$$
\frac{d^{2} x}{d t^{2}}+\mu\left(x^{2}-1\right) \frac{d x}{d t}+x=0
$$

which studied in detail the case $f(x)=x^{2}-1$. Moreover, the generalization was informal and mathematical, and naturally arose from the nonlinear damping of vibrations in electrical circuits considered by Liénard. Setting $d x / d t=z$, Liénard wrote equation (1.1) in the following form of the system of differential equations of first order

$$
\frac{d x}{d t}=z, \quad \frac{d z}{d t}=-x-f(x) z
$$

But in his proof of the uniqueness of a periodic solution of equation (1.1), Liénard used other system of differential equations which is equivalent to system (1.3). For this, in system (1.3) he changed the variable $z=y-F(x)$, where

$$
F(x)=\int_{0}^{x} f(\xi) d \xi,
$$

and obtained the system

$$
\frac{d x}{d t}=y-F(x), \quad \frac{d y}{d t}=-x
$$

Equation (1.1) is referred to as a Liénard equation, and both systems of equations (1.3) and (1.5) are called Liénard systems.

Consider the following differential equation

$$
\frac{d^{2} x}{d t^{2}}+f(x) \frac{d x}{d t}+g(x)=0
$$

which is a generalization of equation (1.1). These equations were obtained by Levinson and Smith [6] in 1942. Equation (1.6) as well as equation (1.1) most of authors call the Liénard equation ${ }^{1}$. The differential equation (1.6) have been studied in many papers $[1,9,5,2,3,11]$. Equation (1.6) one can write in the form of the system of ordinary differential equations

$$
\frac{d x}{d t}=y, \quad \frac{d y}{d t}=-f(x) y-g(x) .
$$

This system can model mechanical systems, where $f(x)$ is known as the damping term and $g(x))$ is called the restoring force or stiffness. System (1.7) is also used to model resistor inductor capacitor circuits with nonlinear circuit elements.

In papers $[4,10,15,13]$ the authors obtained conditions, under which system (1.7) or the equivalent system

$$
\frac{d x}{d t}=z-F(x), \quad \frac{d z}{d t}=-g(x)
$$

has a periodic solution.

The aim of this paper is to obtain other sufficient conditions of the existence of a periodic solution of system (1.7).

\footnotetext{
${ }^{1}$ Some authors call equation (1.6) the generalized Liénard equation.
} 


\section{On the existence of periodic solutions of system (1.7)}

Let us find the conditions that ensure the existence of periodic solutions of system (1.7). Note that the periodic solution of system (1.7) exists if and only if there is a periodic solution of system (1.8). The following theorem gives sufficient conditions for the existence of periodic solutions of system (1.8).

Theorem 2.1. Suppose that $F(x)$ is continuously differentiable, $g(x)$ is locally Lipschitz, and besides

- $x g(x)>0$ for $x \neq 0$;

- the equation $F(x)=0$ has three real roots: $x=b_{1}>0, x=b_{2}<0$, and $x=0$; $F(x)>0$ for $x \in\left(b_{2}, 0\right) \cup\left(b_{1},+\infty\right) ; F(x)<0$ for $x \in\left(-\infty, b_{2}\right) \cup\left(0, b_{1}\right)$;

- $F(x)$ monotonically increases in the intervals $\left(-\infty, b_{2}\right)$ and $\left(b_{1},+\infty\right)$; $F(x) \rightarrow+\infty$ as $x \rightarrow+\infty, F(x) \rightarrow-\infty$ as $x \rightarrow-\infty$.

Then system (1.8) has a nontrivial (nonzero) periodic solution.

Proof. As has been shown in [3, 14], any solution of system (1.8) is a clockwise rotation around the origin, i.e. any solution that starts on the positive semiaxis of ordinate $O z$, sequentially passes the first quadrant, then the fourth, third, second, first again, and so on. Consider the trajectory $x(t), z(t)$ of system (1.8) in the plane $O x z$ starting at the point $H$ with the coordinates $\left(0, z_{H}\right)$ at the zero moment of time $t$ (see Fig. 1 ).

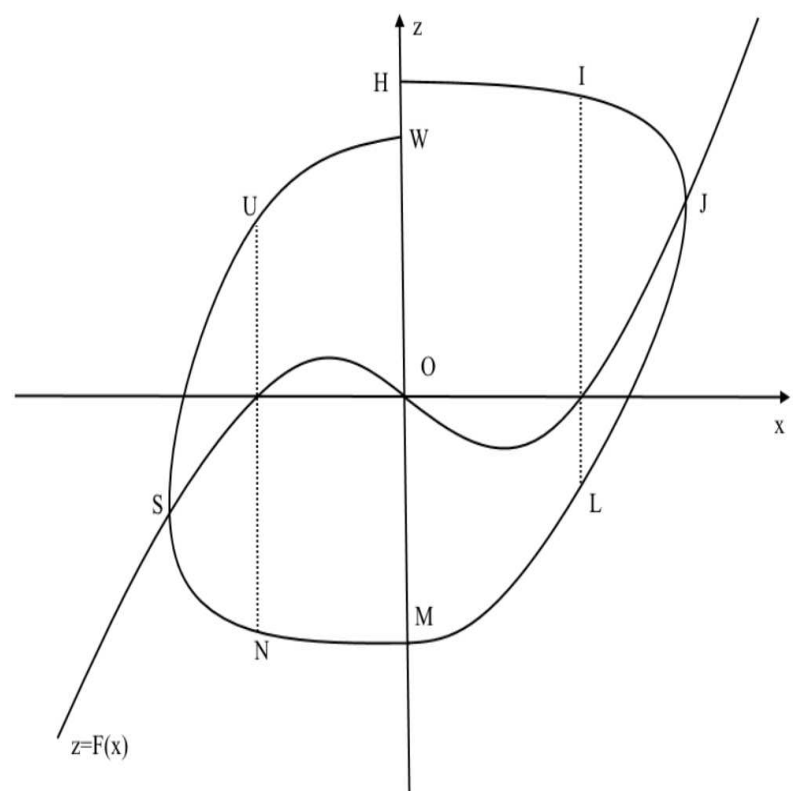

Figure 1

Denote by $J$ and $S$ the points of intersection of this trajectory with the curve $z=F(x)$, by $I$ and $L$ the points of intersection of the trajectory with the straight line 
$x=b_{1}$, by $U$ and $N$ the points of intersection of the trajectory with the straight line $x=b_{2}$, and, finally, by $W$ and $M$ the points of intersection of the trajectory $x(t), z(t)$ with the axis $O z$.

Obviously, the solution $x(t), z(t)$ is periodic if and only if the points $H$ and $W$ coincide, i.e. $z_{H}=z_{W}$.

Denote

$$
G(x):=\int_{0}^{x} g(\xi) d \xi
$$

Consider the function

$$
v(x, z)=\frac{z^{2}}{2}+G(x)
$$

Its derivative along solutions of system (1.8) is equal

$$
\frac{d v(x(t), z(t))}{d t}=-z(t) g(x(t))+g(x(t))[z(t)-F(x(t))]=-g(x(t)) F(x(t)) .
$$

The change of the function $v$ from point $H$ to point $W$ is equal to

$$
\Delta v=v\left(0, z_{W}\right)-v\left(0, z_{H}\right)=\int_{0}^{\tau} \frac{d v(x(t), z(t))}{d t} d t=-\int_{0}^{\tau} g(x(t)) F(x(t)) d t
$$

where $\tau$ is moment of time when the trajectory $x(t), z(t)$ reaches the point $W$. Assume that $z_{H}$ is sufficiently large, such that $x_{J}>b_{1}, x_{S}<b_{2}$. Let us show that $\Delta v$ is a decreasing function of $z_{H}$. To do this, we break the trajectory between $H$ and $W$ into 6 pieces, where the first piece is a segment of the trajectory between points $H$ and $I$, the second piece is a segment of the trajectory between points $I$ and $L$, the third piece is the segment of the trajectory between the points $L$ and $M$, the fourth piece is the segment of the trajectory between the points $M$ and $N$, the fifth piece is a segment of the trajectory between the points $N$ and $U$, the sixth piece is a segment of the trajectory between the points $U$ and $W$. So $\Delta v$ can be represented in the form $\Delta v=\sum_{i=1}^{6} \Delta v_{i}$ where $\Delta v_{i}$ is the change of the function $v$ on $i$-th piece of the trajectory. On the first, third, fourth and sixth pieces, $z$ can be represented as a function of a variable $x$, because on these pieces $x(t)$ either monotonically increases or monotonically decreases; hence, the change of variable $d t=\frac{d x}{z-F(x)}$ is quite correct.

On the second and fifth pieces we use the substitution $d t=-\frac{d z}{g(x)}$. We want to argue that $\Delta v$ is a monotonically decreasing function of $z_{H}$. So consider two trajectories starting at $t=0$ from points $\left(0, z_{H}\right)$ and $\left(0, z_{H}+\Delta z_{H}\right)$, where $\Delta z_{H}>0$. We denote the trajectories of system (1.8), starting at $t=0$ from the points $\left(0, z_{H}\right)$ and $\left(0, z_{H}+\Delta z_{H}\right)$ by symbols $T 1$ and $T 2$ respectively. By virtue of the conditions of the theorem of existence and uniqueness of solutions of system (1.8), trajectories $T 1$ and $T 2$ have no common points, hence, the trajectory $T 2$ is located outside of the trajectory $T 1$, i.e. any ray emerging from the origin, first intersects the trajectory $T 1$ and then the trajectory $T 2$. Let us discover how changes the expression for $\Delta v_{i}(i=1, \ldots, 6)$ in the transition from the trajectory $T 1$ to the trajectory $T 2$.

$$
\Delta v_{1}=\int_{0}^{b_{1}} \frac{g(x)[-F(x)]}{z(x)-F(x)} d x=\int_{0}^{b_{1}} \frac{g(x)|F(x)|}{|z(x)-F(x)|} d x .
$$


The value for $z(x)$ on the trajectory $T 2$ is more then the value for $z(x)$ on $T 1$, hence, $\Delta v_{1}(T 2)<\Delta v_{1}(T 1)$. Here and below $\Delta v_{i}(T 2)$ and $\Delta v_{i}(T 1)$ denote the values of $\Delta v_{i}$ on trajectories $T 2$ and $T 1$ respectively.

$$
\Delta v_{2}=-\int_{z_{I}}^{z_{L}} g(x) F(x)\left[-\frac{d z}{g(x)}\right]=-\int_{z_{L}}^{z_{I}} F(x(z)) d z .
$$

Taking into account that on this piece $F(x)$ is positive and monotonically increasing and $\left.x(z)\right|_{T 2}>\left.x(z)\right|_{T 1}$, we obtain that $\Delta v_{2}(T 2)<\Delta v_{2}(T 1)$.

$$
\Delta v_{3}=\int_{b_{1}}^{0} \frac{g(x)[-F(x)]}{z(x)-F(x)} d x=\int_{0}^{b_{1}} \frac{g(x)|F(x)|}{|z(x)-F(x)|} d x
$$

In this case we also have $\Delta v_{3}(T 2)<\Delta v_{3}(T 1)$.

$$
\Delta v_{4}=\int_{0}^{b_{2}} \frac{[-g(x)] F(x)}{z(x)-F(x)} d x=\int_{b_{2}}^{0} \frac{[-g(x)] F(x)}{F(x)-z(x)} d x
$$

whence $\Delta v_{4}(T 2)<\Delta v_{4}(T 1)$.

$$
\Delta v_{5}=-\int_{z_{N}}^{z_{U}} g(x) F(x)\left[-\frac{d z}{g(x)}\right]=\int_{z_{N}}^{z_{U}} F(x(z)) d z .
$$

On this piece $F(x)$ is negative. Since $\left.x(z)\right|_{T 2}<\left.x(z)\right|_{T 1}$, then

$$
\left.F(x(z))\right|_{x(z) \in T 2}<\left.F(x(z))\right|_{x(z) \in T 1},
$$

hence $\Delta v_{5}(T 2)<\Delta v_{5}(T 1)$.

$$
\Delta v_{6}=-\int_{b_{2}}^{0} \frac{g(x) F(x)}{z(x)-F(x)} d x=\int_{b_{2}}^{0} \frac{[-g(x)] F(x)}{z(x)-F(x)} d x .
$$

Here $\left.z(x)\right|_{T 2}>\left.z(x)\right|_{T 1}$, therefore $\Delta v_{6}(T 2)<\Delta v_{6}(T 1)$. Thus it has been proved that $\Delta v_{i}(i=1, \ldots, 6)$ decrease if $z_{H}$ increase, hence $\Delta v$ also decreases with increasing $z_{H}$. Let us show that

$$
\lim _{z_{H} \rightarrow+\infty} \Delta v=-\infty
$$

To do this, it is enough to prove that

$$
\lim _{z_{H} \rightarrow+\infty} \Delta v_{2}=-\infty
$$

We will show that $z_{I}$ increases indefinitely with unlimited increase of the value $z_{H}$. Getting rid of $t$ in system (1.8) and passing to the argument $x$, we write the differential equation which describes the orbit $H I J$ :

$$
\frac{d z}{d x}=-\frac{g(x)}{z-F(x)} .
$$

According to the condition of the theorem $F(x)<0$ for $x \in\left(0, b_{1}\right)$, hence

$$
\frac{g(x)}{z-F(x)}<\frac{g(x)}{z} \text { for } x \in\left(0, b_{1}\right) \text {. }
$$


From equation (2.3) and inequality (2.4) it follows

$$
-\frac{d z}{d x}<\frac{g(x)}{z} \text { for } x \in\left(0, b_{1}\right) .
$$

Separating variables and integrating, we obtain

$$
\frac{1}{2} z^{2}\left(b_{1}\right)-\frac{1}{2} z_{H}^{2}>-\int_{0}^{b_{1}} g(x) d x,
$$

whence bearing in mind that $z\left(b_{1}\right)=z_{I}$, we get that $z_{I} \rightarrow+\infty$ if $z_{H} \rightarrow+\infty$.

Let $c \in\left(b_{1}, x_{J}\right)$. Let us designate the ordinates of the intersection points of the trajectory $T 1$ and the line $x=c$ on pieces $I J$ and $J L$, respectively $z^{*}$ and $z^{* *}$. Taking into account that $L$ is the intersection point of the trajectory $T 1$ and the line $x=b_{1}$, we conclude that $z_{L}<0$ (see Fig.1). Bearing in mind the continuity of the trajectory $T 1$, the value $c \in\left(b_{1}, x_{J}\right)$ we choose so close to the value of $b_{1}$ that $z^{* *}<0$.

Let $z(x)$ be the solution of equation (2.3) such that $z(0)=z_{H}$. We shall show that $z(c) \rightarrow+\infty$ if $z_{H} \rightarrow+\infty$. The inequality $z-F(x)>z-F(c)$ holds on the interval $\left(b_{1}, c\right)$ because the function $F(x)$ monotonically increases on this interval. Hence equation (2.3) yields

$$
-\frac{d z}{d x}=\frac{g(x)}{z-F(x)}<\frac{g(x)}{z-F(c)} .
$$

Separating variables and integrating, we obtain

$$
-\left[\frac{1}{2} z^{2}-F(c) z\right]_{z_{I}}^{z(c)}<\int_{b_{1}}^{c} g(x) d x,
$$

whence (taking into account that $z(c)=z^{*}>0$ ) it follows the inequality

$$
z(c)>F(c)+\sqrt{\left[z_{I}-F(c)\right]^{2}-2 \int_{b_{1}}^{c} g(x) d x} .
$$

Since $z_{I} \rightarrow+\infty$ if $z_{H} \rightarrow+\infty$, then $z^{*}=z(c) \rightarrow+\infty$ if $z_{H} \rightarrow+\infty$.

Bearing in mind that $F(x)$ increases for $x>b_{1}$, we have

$$
\begin{aligned}
\Delta v_{2} & =-\int_{z_{L}}^{z_{I}} F(x(z)) d z<-F(c)\left(z^{*}-z^{* *}\right) \\
& <-F(c)\left[F(c)+\sqrt{\left[z_{I}-F(c)\right]^{2}-2 \int_{b_{1}}^{c} g(x) d x}\right] .
\end{aligned}
$$

The obtained inequality implies that $\Delta v_{2} \rightarrow-\infty$ if $z_{H} \rightarrow+\infty$.

If we choose $z_{H}$ small enough, such that the entire trajectory between points $H$ and $W$ is located in the domain $x \in\left(b_{2}, b_{1}\right)$, then obviously that $\Delta v>0$. Taking into account that $\Delta v$ tends to $-\infty$ when $z_{H} \rightarrow+\infty$, one can conclude that there exists a value $z_{H}>0$ such that $\Delta v=0$. This means that there exists a periodic solution of system (1.8). The proof is comlpete.

Remark 2.2. If additionally to conditions of the theorem, one of the following conditions 
- $G\left(a_{1}\right)=G\left(a_{2}\right)$ where $a_{1}$ and $a_{2}$ are positive and negative roots of equation $f(x)=0$, and $G( \pm \infty)=+\infty$

- $f(x)$ is even, $g(x)$ is odd, $G(+\infty)=+\infty$,

is satisfied, then equation (1.7) has a single periodic solution [14].

\section{References}

[1] Albarakati, W.A., Lloyd, N.G., Pearson, J.M., Transformation to Liénard form, Electronic Journal of Differential Equations, 76(2000), 1-11.

[2] Bowman, T.T., Periodic solutions of Liénard systems with symmetries, Nonlinear Analysis, 2(4)(1978), 457-464.

[3] Carletti, T., Villari, G., A note on existence and uniqueness of limit cycles for Liénard systems, Journal of Mathematical Analysis and Applications, 307(2005), no. 2, 763-773.

[4] Filippov, A.F., A sufficient condition for the existence of a stable limit cycle for the equation of the second order, (in Russian), Sbornik: Mathematics, 30(1952), no. 1, 171180.

[5] Ignat'ev, A.O., Kirichenko, V.V., On necessary conditions for global asymptotic stability of equilibrium for the Liénard equation, Mathematical Notes, 93(2013), no. 1-2, 75-82.

[6] Levinson, N., Smith, O., A general equation for relaxation oscillations, Duke Mathematical Journal, 9(1942), no. 2, 382-403.

[7] Liénard, A., Étude des oscillations entreténues, Revue Générale de L'électricité, 23(1928), 901-912.

[8] Liénard, A., Étude des oscillations entreténues, Revue Générale de L'électricité, 23(1928), 946-954.

[9] Lynch, S., Christopher, C.J., Limit cycles in highly non-linear differential equations, Journal of Sound and Vibration, 224(1999), no. 3, 505-517.

[10] Neumann, D.A., Sabbagh, L., Periodic solutions of Liénard systems, Journal of Mathematical Analysis and Applications, 62(1978), no. 1, 148-156.

[11] Odani, K., Existence of Exactly $N$ Periodic Solutions for Liénard Systems, Funkcialaj Ekvacioj, 39(1996), 217-234.

[12] van der Pol, B., On relaxation-oscillations, The London, Edinburgh and Dublin Philosophical Magazine and Journal of Science, 2(1927), 978-992.

[13] Sabatini, M., On the period function of Liénard systems, Journal of Differential Equation, 152(1999), 467-487.

[14] Sabatini, M., Villari, G., On the uniqueness of limit cycles for Liénard equation: the legacy of G. Sansone, Matematiche (Catania), 65(2010), no. 2, 201-214.

[15] Sugie, J., Hara, T., Non-existence of periodic solutions of the Liénard system, Journal of Mathematical Analysis and Applications, 159(1991), no. 1, 224-236.

Alexander Ignatyev

Institute of Applied Mathematics and Mechanics,

R. Luxemburg Street, 74,

Donetsk-83114, Ukraine

e-mail: aoignat@mail.ru 